\title{
Estimation of spleen volume and surface area of the newborns' cadaveric spleen using stereological methods
}

\author{
A. Sağıroğlu1 , N. Acer², T. Ertekin², E. Kurtoğlu², A. Coşkun³, A. Yıldırım³ G. Zararsız \\ ${ }^{1}$ Department of Anatomy, Kayseri Education and Research Hospital, Kayseri, Turkey \\ 2Department of Anatomy, Erciyes University Faculty of Medicine, Kayseri, Turkey \\ ${ }^{3}$ Department of Radiology, Erciyes University Faculty of Medicine, Kayseri, Turkey \\ ${ }^{4}$ Department of Biostatistics and Medical Informatics, Erciyes University Faculty of Medicine, Kayseri, Turkey
}

[Received 13 August 2013; Accepted 2 October 2013]

Background: The purpose of this study was to compare different techniques for the estimation of spleen volume and surface area using magnetic resonance imaging (MRI) images, ultrasonography (USG) images and cadaveric specimen, and to evaluate errors associated with volume estimation techniques based on fluid displacement.

Materials and methods: Five newborn cadavers, aged $39.7 \pm 1.5$ weeks, weighted $2.220 \pm 1.056 \mathrm{~g}$, were included in the present study. Three different methods were used to assess the spleen volume. The vertical section technique was applied using cycloid test probes for estimation of spleen surface area in MRI.

Results: The mean \pm standard deviation of spleen volumes by fluid displacement was $4.82 \pm 3.85 \mathrm{~cm}^{3}$. Volumes determined by the Cavalieri's principle using physical section and point-counting techniques were $4.45 \pm 3.47 \mathrm{~cm}^{3}$ and $4.65 \pm 3.75 \mathrm{~cm}^{3}$, respectively; volumes measured by USG and cadaver using ellipsoid formula were $4.70 \pm 3.02 \mathrm{~cm}^{3}$ and $5.98 \pm 4.58 \mathrm{~cm}^{3}$, respectively. No significant differences were found among all methods ( $p>0.05)$. The spleen surface area was calculated as a $32.3 \pm 20.6 \mathrm{~cm}^{2}$ by physical sections using cadaver and also it was determined on axial, sagittal and coronal MR planes as $24.9 \pm 15.2 \mathrm{~cm}^{2}$, $18.5 \pm 5.92 \mathrm{~cm}^{2}$ and $24.3 \pm 12.7 \mathrm{~cm}^{2}$, respectively.

Conclusions: As a result, MR images allow an easy, reliable and reproducible volume and surface area estimation of normal and abnormal spleen using Cavalieri's principle. We consider that our study may serve as a reference for similar studies to be conducted in future. (Folia Morphol 2014; 73, 2: 183-192)

Key words: spleen volume, surface area, stereology, Cavalieri's principle

\section{INTRODUCTION}

The spleen is an important lymphoid organ with both immunological and haematopoietic functions and also the largest of the secondary lymphoid organs [16].
Changes in human spleen size and morphology take an important place in clinical diagnosis and treatment. It is possible to reach an overall idea about the size of the spleen through palpation and percussion, however, these methods may sometimes become in- 
adequate, because spleen is not usually palpable till it enlarges 2 to 3 times its own size $[4,14]$. The size and weight of the spleen may vary according to an underlying pathology or age. In certain pathological situations, such as leukaemia, the spleen assists in the reformation of blood. Due to blood formation in areas outside of the bone marrow, the spleen is exposed to myeloid metaplasia. This leads to the development of splenomegaly. Splenomegaly is an important clinical sign for diagnosing many diseases such as malaria, collagen tissue diseases, portal hypertension, glycogen storage disorder, neoplastic blood diseases and other disorders $[4,16]$.

The aetiology of newborn splenic size enlargement is unclear. The foetal spleen has immunological and haematological functions, so mechanisms in these both categories should be considered [6]. Foetal splenomegaly could also result from haematological mechanisms. Erythropoiesis in the spleen normally ceases before birth, but may resume in some pathological conditions, such as haemolytic anaemia [35].

Different techniques, which are used for the estimation of spleen volume by ultrasonography (USG) $[13,17,32]$, magnetic resonance imaging (MRI) [34], and computed tomography (CT) [36] have been presented in literature. In addition, Asghar et al. [4] used surface rendering technique of 3-D-Doctor by analyses of $C T$ images, and then calculated the volume and the surface area of the spleen. Mazonakis et al. [21] estimated splenic volume using random marking technique on MR images. Splenic volume had been estimated by USG, however, some studies showed that it can be inaccurate because of the variable and irregular contour of spleen and overlapping of splenic outline by bone, bowel gas, or left kidney $[4,15]$.

The acquisition and analysis of medical images in accordance with the rules of modern design-based stereology $[27,30]$ enable precise and unbiased estimates of geometric quantities, such as volume and surface area, to be obtained in a moderate workload.

Using the Cavalieri's principle, the estimation of an organ volume can be performed with mathematical unbiasedness and free from any systematic error [11, 25]. The mentioned method was first described by an Italian mathematician Bonaventura Cavalieri. He proposed that volume of the irregular shaped objects could be estimated from a set of 2-dimensional (2D) slices through the object, provided that they are parallel, separated by a known distance, and begin randomly within the object. These criteria are met by standard CT and MR images [36]. The relevant method has been refined and applied in a series of studies using different scanning techniques $[11,17]$. Some studies have proven this estimator to be as accurate as digitisation-based methods and to correlate closely with displacement volume measurements [2].

Few studies were found in literature on estimation of spleen volume and surface area in newborns' cadavers based on MR and USG measurements [4]. There is no study which investigated the spleen volume using gold standard method in newborn cadavers.

The aim of this study was to estimate the spleen volume by Archimedes' principle, point-counting method, and ellipsoid formula using USG, MRI, and cadaver specimens, and also to estimate the surface area of the spleen using vertical section technique from newborn cadavers, and thereby to determine the differences in measurement observed among these methods.

\section{MATERIALS AND METHODS}

Five newborn cadavers, kept for practical anatomy education, from the Erciyes University Medical Faculty Department of Anatomy laboratory, were used in this study. Erciyes University Medical Faculty Ethics Committee approval was obtained prior to commencing the study. Cadavers with no morphological spleen defects were included in the study. Full abdomen USG and MRI procedures were performed to obtain spleen images from cadavers before the dissection of the spleen.

First, cadavers were fixed by neck vessels perfusion according to their sizes and then kept in $10 \%$ formalin solution during the study period (approximately 6 months). Each spleen was extracted from the abdomen, examined, and photographed externally with a digital camera. The following 3 methods were used for the estimation of spleen volume.

\section{Archimedes' volume as a reference volume}

By placing the removed spleen into beakers containing water, the actual spleen volumes were calculated according to the Archimedes' principle (i.e. according to the amount of water displacement). Archimedes' principle was used to obtain an independent determination of the spleen volume. Water displaced by spleens was measured using a graduated cylinder to determine the spleen volume. The measurements were compared to the other methods and the mean and standard deviation ( \pm SD) of the measurements were calculated. 


\section{Volume estimation with USG measurements}

USG imaging was performed according to the following protocol: with the sonosite M-Turbo portable USG device, measurements were performed using the HFL $38 \times / 13-6 \mathrm{MHz}$ linear transducer or $\mathrm{C} 60 \times / 5-2 \mathrm{MHz}$ convex transducer probes.

All USG examinations were performed by a single radiologist $(A C)$. The measurements were performed on the coronal plane that included the splenic hilum, with the cadaver maintained in the slight right lateral decubitis.

The length, width and thickness of each spleen were measured using USG. The largest longitudinal distance between the dome and the tip of the spleen (the distance between the posterior extremity and anterior extremity) was measured. This value was considered as a measure of length. The thickness (the distance between visceral surface and diaphragmatic surface) and the width (the distance between inferior border and superior border) were measured in the coronal plane USG image. Additionally, the length, width and thickness of each spleen were measured using a caliper on cadaver specimen (Fig. 1A, B).

Volumes were calculated according to the ellipsoid formula using USG images and caliper measurements. The ellipsoid formula is shown below:

Volume $=$ Lenght $\times$ Widht $\times$ Thickness $\times 1 / 2$

\section{Volume estimation using stereological method}

Using the Cavalieri's method, an estimate of the volume of a structure of an arbitrary shape and size may be obtained efficiently and precisely. The volume estimation using Cavalieri's method can be expressed as a following equation (Eq. 2) [30]:

$$
V=T \times \sum_{i=1}^{n} V_{i}
$$

where $V_{i}$ is the total volume of tissue slice (which may comprise several slice profiles) in the ith slab and $T$ is the slice thickness.

We have estimated spleen volume using MR images and cadaver slices of spleen. MRI was performed according to the following protocol: morphometric MRI evaluation was performed using a $1.5 \mathrm{~T}$ magnet (Philips, Interna, Bes) device. On the T1 weighted axial plan, 2D FLASH (Fast Low Angle Shot) sequence was obtained for the surface area measurement, and the following technical factors were employed: TR (repetition time) $=168 \mathrm{~ms}$, TE (echo time) $=4.1 \mathrm{msn}$, FOV (field of view) $=25 \mathrm{~cm}$, matrix $=128 \times 256$,

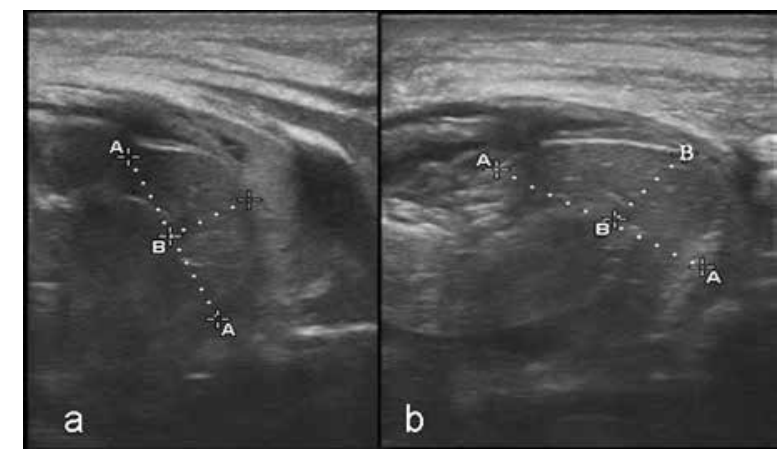

Figure 1. Measurement of length $(A-A)$ of the spleen with ultrasonography (USG) (a); measurement of thickness ( $A-A)$ and width (B-B) values of the spleen with USG (b).

flip angle $=90$ degrees, voxel size $=8 \times 8 \times 8 \mathrm{~mm}$, slice thickness $=1.6 \mathrm{~mm}$, and gap $=0$.

The obtained images were viewed on a computer using the Hipax Patient CD Viewer V.1.6.6 program. MR images of $1.6 \mathrm{~mm}$ thickness for the 3 planes (axial, coronal and sagittal) were examined by a radiologist.

\section{Point-counting method}

Point-counting method is based on the Cavalieri's principle. According to this method, the MR images of a section series of $1.6 \mathrm{~mm}$ thickness were used to estimate the volume of the structures. The films were saved on a computer and the transparent square grid test system with $\mathrm{d}=2.5 \mathrm{~mm}$ between test points was superimposed, randomly covering the entire image frame.

In addition, the spleens were sliced at $0.5 \mathrm{~cm}$ intervals using a special slicing device. Volume calculations were performed on each individual spleen slice by using the transparent square grid test system with $d=2.5 \mathrm{~mm}$ between test points (Fig. 2A, B).

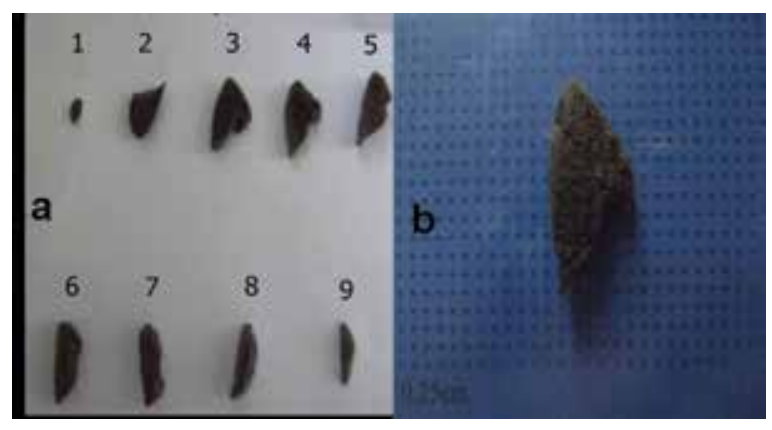

Figure 2. Sections which were sliced by fractitioner knife (a); random section taken on the point-counting grid (b). 
The points hitting the structure sectioned surface area (MR images and cadaver slice of spleen) were counted for each section and the volume of the spleen was estimated using the following formula for both of the specimens (MR images and cadaver slice of spleen) [1]:

$$
V(P C)=T \times[d]^{2} \times \sum p
$$

where $T$ is the section thickness, $d$ is the distance between the test points of the grid, and $p$ is the total number of points hitting the sectioned sliced surface areas of the structure. According to this volumetric technique, a square grid of test points was positioned on each MR image, and all points covering the structure were counted.

\section{Estimation of spleen surface area using vertical section method}

The stereological method of surface area estimation using vertical sections produces unbiased estimates of surface area. In this technique, the surface is sampled at systematic random positions in 3-D. The method combines the Cavalieri's principle with vertical sectioning [5].

The vertical section techniques generally use the cycloid test probes for estimation of the surface area. The relevant formula for estimating the surface area from an exhaustive series of vertical sections was calculated using equation $4[1,30]$ :

$$
S=2 \times T \times a / l \times l
$$

We have calculated the surface area of spleen on MR images and cadaver slice of spleen using vertical sections.

The equation 4 was used for the surface area estimations of spleen on cadaver slices. Then, we have modified the above formula (Eq. 4) for the surface area estimations of radiological images shown below:

$$
S=\frac{2}{n} \times T \times\left(\frac{a / l \times S U}{S L}\right) \times I
$$

where $n$ is the number of random systematic orientation, $a / l$ is the ratio of the test area to the cycloid length, $T$ is the distance between serial sections, $S U$ is the scale unit of the printed film, $S L$ is the measured length of the scale printed on the film and $l$ is the number of intersections on all sections.

We have used 3 orientations to calculate the a/l using $\mathrm{d}=0.55 \mathrm{~cm}$. In our experiment, cycloid test lines were found to give a $1.727 \mathrm{~cm}$ ratio of area associated with each cycloid for a/l according to Eq. 6 (Fig. 3):

$$
a / l=\pi \times d / 2
$$

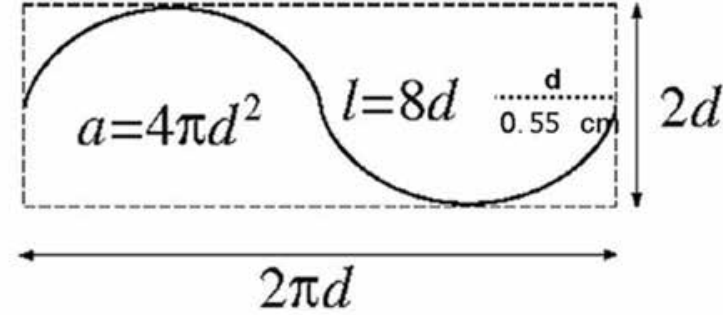

Figure 3. Cycloid probe.

The coefficient of error (CE) is given in Eq. 7 [10]. The $\mathrm{CE}$ of volume is calculated as follows. It can be shown that:

$$
C E^{2}(V)=C E_{C A V}^{2}(V)+C E_{P C}^{2}
$$

where $C E^{2}(V)$ is $C E$ of the volume estimate, $C E^{2}{ }_{P C}(V)$ is true mean variability due to point-counting within sections, $C E^{2}{ }_{C A V}(V)$ is true contribution of the variability among sections. In Eq. 7, $C E^{2}(V)$ is the square coefficient of error of the estimator of $V$ when the areas are measured exactly. The CE value is acceptable lower than $5 \%$ according to the literature [33]. The CE of the surface area is computed as follows:

$C E=\left(\sum_{i=1}^{n}\right)^{-1} \times\left[\frac{1}{12}\left(3 \sum_{i=1}^{n} A_{i}^{2}+\sum_{i=1}^{n-2} A A_{i+2}-4 \sum_{i=1}^{n-1} A A_{i+1}\right)\right]^{1 / 2}$

In this study, we have calculated the CE values as predictive, using the $\mathrm{R}$ program. First, by using the statistical package $R$, codes were developed to calculate the contribution to the predictive $\mathrm{CE}$.

\section{Statistical analysis}

To assess the agreement between the volume measurements of Archimedes' method and other methods, statistical agreement measures, including concordance correlation coefficient (CCC), intraclass correlation coefficient (ICC), Pearson's correlation coefficient $(r), p$ values obtained from paired-t test and least square analysis $\left(p_{p t}, p_{l s}\right)$ and coefficient of variation, were calculated. For the complete agreement, CCC, ICC and $r$ values should be maximised and $\mathrm{p}_{\mathrm{pt}}, \mathrm{p}_{\mathrm{ls}}$ and coefficient of variation (CV) values should be minimised. Details about these measures can be found in reference No. 18 [18]. The analyses were conducted using R 2.15 .0 software.

To assess the agreement between physical section and other surface areas, statistical agreement measures, including $\mathrm{CCC}, \mathrm{ICC}, \mathrm{r}, \mathrm{p}_{\mathrm{pt}} \mathrm{p}_{\mathrm{Is}}$ and coefficient of variation, were calculated. For the complete agreement, CCC, ICC, and $r$ values should be 
Table 1. Measurement of the spleen using ultrasonography (USG) and caliper

\begin{tabular}{lcccccccc}
\hline Cadaver No. & \multicolumn{3}{c}{ USG [cm] } & & \multicolumn{3}{c}{ Caliper [cm] } \\
\cline { 2 - 3 } & Length & Width & Thickness & & Length & Width & Thickness \\
\hline 1 & 4.74 & 3.06 & 1.23 & & 5.20 & 1.10 & 2.60 \\
2 & 2.99 & 0.95 & 1.48 & & 2.40 & 1.30 & 1.20 \\
3 & 2.31 & 2.38 & 1.03 & & 2.60 & 2.50 & 1.20 \\
4 & 2.31 & 2.40 & & 0.98 & & 2.80 & 1.50 & 1.30 \\
5 & 4.14 & 2.83 & 1.02 & & 4.10 & 3.10 & 2.00 \\
Mean \pm SD & $3.29 \pm 1.09$ & $2.32 \pm 0.82$ & $1.15 \pm 0.21$ & & $3.42 \pm 1.19$ & $2.16 \pm 0.81$ & $1.42 \pm 0.35$ \\
\hline
\end{tabular}

Table 2. Comparison of magnetic resonance imaging (MRI) volumes with the principle of Archimedes (gold standard). Values in parentheses indicate the differences among the volumes obtained by Archimedes (gold standard) and the estimated MRI volumes by using point-counting technique

\begin{tabular}{lcccc}
\hline Cadaver No. & Archimedes & \multicolumn{3}{c}{ MRI volumes [cm ${ }^{3}$ ] } \\
\cline { 3 - 4 } & & Axial & Sagittal & Coronal \\
\hline 1 & 9.00 & $6.69(-2.21)$ & $7.22(-1.78)$ & $10.1(1.10)$ \\
2 & 1.60 & $1.80(0.20)$ & $1.60(+0.00)$ & $2.58(0.98)$ \\
3 & 2.90 & $2.13(-0.77)$ & $2.91(+0.01)$ & $1.88(-1.02)$ \\
4 & 1.60 & $2.30(0.70)$ & $0.63(-0.97)$ & $1.70(0.10)$ \\
5 & 9.00 & $7.78(-1.12)$ & $10.50(1.50)$ & $10.0(1.00)$ \\
Mean \pm SD & $4.82 \pm 3.85$ & $4.14 \pm 2.86(-0.68 \pm 1.19)$ & $4.57 \pm 4.16(-0.25 \pm 1.13)$ & $5.25 \pm 4.39(0.43 \pm 0.91)$ \\
\hline
\end{tabular}

maximised and $p_{p t}, p_{l s}$ and $C V$ values should be minimised. Details about these measures can be found in reference No. 18 [18]. The analyses were conducted using R 2.15.0 software.

\section{RESULTS}

In our study, size measurements were performed on the spleen from newborn cadavers by using USG and calipers, after stereological measurements were performed on the MR images and cadaver slices of spleen. USG and caliper measurements are shown in Table 1.

We have estimated that the mean \pm SD spleen volumes obtained with Archimedes were $4.82 \pm 3.85 \mathrm{~cm}^{3}$. Mean volumes determined by the point-counting using MR images and physical section of cadaver specimen were $4.66 \pm 3.76 \mathrm{~cm}^{3}$ and $4.45 \pm 3.46 \mathrm{~cm}^{3}$, respectively. Three plane MR images (axial, coronal and sagittal) were used in the calculation of the mean spleen volume obtained by pointcounting, and these volumes are shown in Table 2. Volumes estimated by ellipsoid formula using USG and caliper measurements were $4.70 \pm 3.02 \mathrm{~cm}^{3}$ and $5.98 \pm 4.58 \mathrm{~cm}^{3}$, respectively (Table 3 ). According to the 3 methods, our results were correlated with each other. There were no significant differences among these methods ( $p>0.05$ ) (Table 4).

Spleen volumes estimated by point-counting using axial, sagittal, and coronal MR images and fluid displacement (the gold standard) differed by between -2.21 and $0.70 \mathrm{~cm}^{3},-1.78$ and $1.50 \mathrm{~cm}^{3},-1.02$ and $1.10 \mathrm{~cm}^{3}$, respectively. We have determined underestimation of spleen volume for axial and sagittal MR images and overestimation for coronal MR images. The mean \pm SD decreases in spleen volume for axial and sagittal MR images were $0.68 \pm 1.19 \mathrm{~cm}^{3}$, $0.25 \pm 1.23 \mathrm{~cm}^{3}$ and the mean increase for coronal MR image was $0.43 \pm 0.91 \mathrm{~cm}^{3}$ (Table 2, Fig. 4).

There was an increase in 2 of 5 subjects for spleen volume estimations obtained by using physical section, although there was a slight decrease in 3 cadavers. The mean \pm SD decrease in splenic volume for all the subjects was $0.37 \pm 0.45 \mathrm{~cm}^{3}$ (Table 3, Fig. 4).

Spleen volumes which were obtained by ellipsoid formula using USG and caliper measurements and fluid displacement (the gold standard) were compared with each other and they differed between -2.76 and $1.22 \mathrm{~cm}^{3},-1.27$ and $4.20 \mathrm{~cm}^{3}$, respectively. We have determined underestimation of spleen volume 
Table 3. Comparison of other methods with the principle of Archimedes (gold standard). Values in parentheses indicate the differences among the volumes obtained by Archimedes (gold standard) and other methods (MRI, USG, physical section, caliper)

\begin{tabular}{lcccccc}
\hline Cadaver No & Archimedes & \multicolumn{2}{c}{ Point-counting } & & \multicolumn{2}{c}{ Ellipsoid formula } \\
\cline { 3 - 4 } \cline { 6 - 7 } & & MRI & Physical section & & USG & Caliper \\
\hline 1 & 9.00 & $8.00(1.00)$ & $8.16(-0.84)$ & & $9.31(0.31)$ & $7.73(-1.27)$ \\
2 & 1.60 & $1.99(-0.39)$ & $1.71(0.11)$ & & $2.19(0.59)$ & $2.10(0.50)$ \\
3 & 2.90 & $2.30(0.60)$ & $2.36(-0.54)$ & & $2.95(0.05)$ & $4.05(1.15)$ \\
4 & 1.60 & $1.54(-0.06)$ & $1.71(0.11)$ & & $2.82(1.22)$ & $2.83(1.23)$ \\
5 & 9.00 & $9.42(-0.42)$ & $8.31(-0.69)$ & & $6.24(-2.76)$ & $13.2(4.20)$ \\
Mean \pm SD & $4.82 \pm 3.85$ & $4.65 \pm 3.75$ & $4.45 \pm 3.47$ & & $4.70 \pm 3.02$ & $5.98 \pm 4.58$ \\
& & $(-0.17 \pm 0.62)$ & $(-0.37 \pm 0.45)$ & & $(-0.12 \pm 1.54)$ & $(+1.16 \pm 1.97)$ \\
\hline
\end{tabular}

Table 4. Statistical agreement measures for axial, sagittal, coronal magnetic resonance imaging (MRI), ultrasonography (USG), physical section, caliper and Archimedes volumes

\begin{tabular}{|c|c|c|c|c|c|c|}
\hline \multirow{2}{*}{$\begin{array}{l}\text { Agreement } \\
\text { measures }\end{array}$} & \multicolumn{3}{|c|}{ MRI $\left[\mathrm{cm}^{3}\right]$} & \multirow{2}{*}{$\begin{array}{l}\text { USG } \\
{\left[\mathrm{cm}^{3}\right]}\end{array}$} & \multirow{2}{*}{$\begin{array}{c}\text { Physical section } \\
{\left[\mathrm{cm}^{3}\right]}\end{array}$} & \multirow{2}{*}{$\begin{array}{l}\text { Caliper } \\
{\left[\mathrm{cm}^{3}\right]}\end{array}$} \\
\hline & Axial & Sagittal & Coronal & & & \\
\hline CCC (95\% CI) & $0.92(0.69-0.98)$ & $0.95(0.64-0.99)$ & $0.97(0.82-1.00)$ & $0.90(0.48-0.98)$ & $0.99(0.94-1.00)$ & $0.85(0.26-0.98)$ \\
\hline ICC (95\% CI) & $0.94(0.53-0.99)$ & $0.95(0.62-1.00)$ & $0.98(0.79-1.00)$ & $0.90(0.33-0.99)$ & $0.99(0.93-1.00)$ & $0.89(0.29-0.99)$ \\
\hline r (95\% Cl) & $0.98(0.73-1.00)$ & $0.96(0.47-1.00)$ & $0.98(0.76-1.00)$ & $0.93(0.25-1.00)$ & $0.99(0.97-1.00)$ & $0.90(0.11-0.99)$ \\
\hline $\mathrm{p}_{\mathrm{pt}}$ & 0.269 & 0.675 & 0.347 & 0.872 & 0.140 & 0.258 \\
\hline $\mathrm{p}_{\mathrm{ls}}$ & 0.003 & 0.011 & 0.002 & 0.023 & $<0.001$ & 0.035 \\
\hline CV (\%) & 19.90 & 16.98 & 12.90 & 20.53 & 8.35 & 27.67 \\
\hline
\end{tabular}

CCC — concordance correlation coefficient; $\mathrm{Cl}$ - confidence interval; ICC — intraclass correlation coefficient; $r$ - Pearson's correlation coefficient; $\mathrm{p}_{\mathrm{pt}}-\mathrm{p}$ value obtained from paired-t test; $p_{\mathrm{ls}}-\mathrm{p}$ value obtained from least square analysis; $C V$ - coefficient of variation; maximum CCC, ICC and $r$ values and minimum $p_{\mathrm{pt}} \mathrm{p}_{\mathrm{ls}}$ and CV values indicate best agreement between physical section and gold standard volume
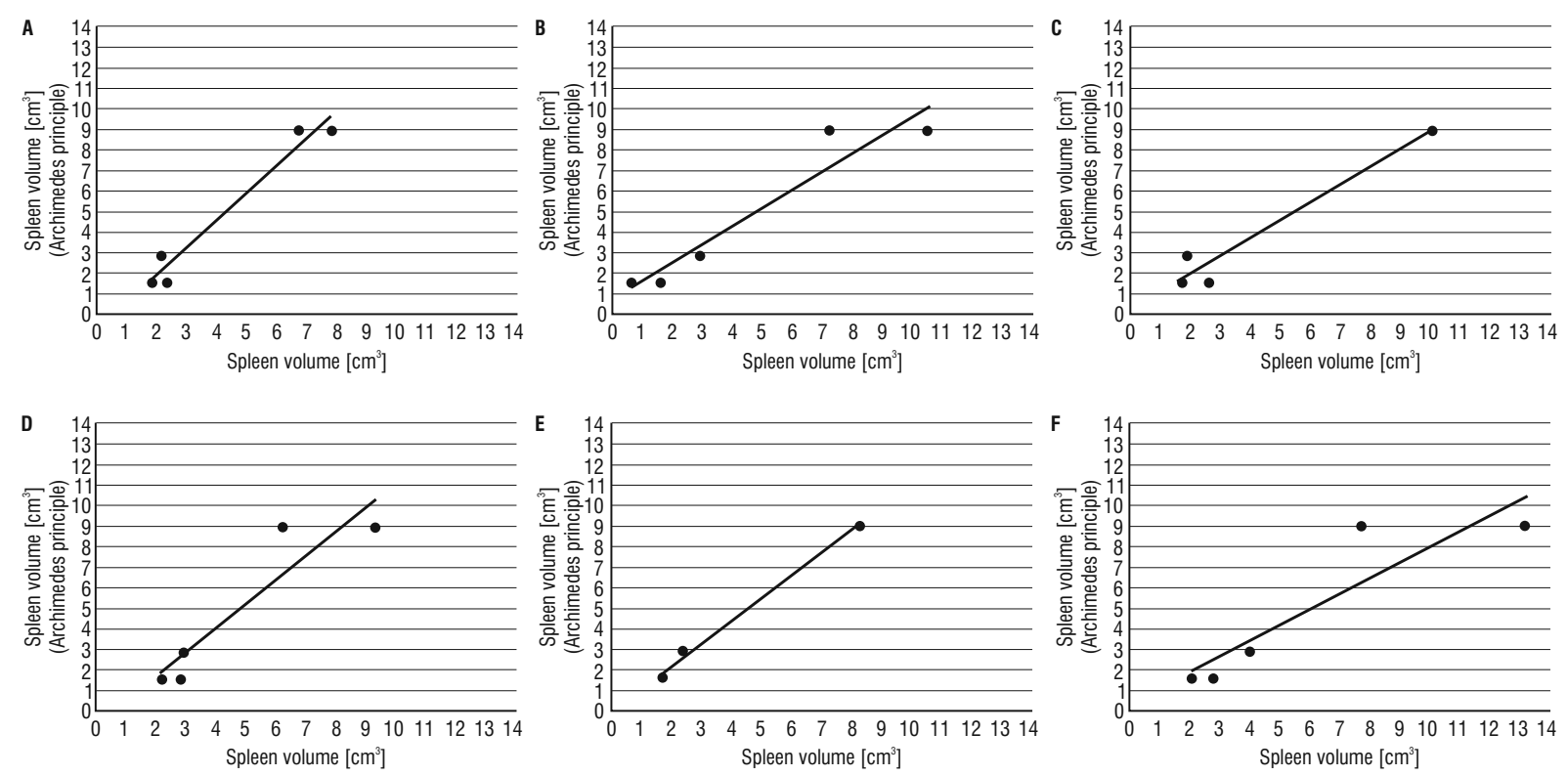

Figure 4. Scatter plots displaying the volume estimates of Archimedes principle versus (A) axial magnetic resonance imaging (MRI), (B) sagittal MRI, (C) coronal MRI, (D) ultrasonography, (E) physical section, (F) caliper. 
Table 5. Comparison of magnetic resonance imaging surface area estimates with physical section (gold standard)

\begin{tabular}{|c|c|c|c|c|c|c|c|}
\hline \multirow[t]{2}{*}{ Cadaver No. } & \multirow{2}{*}{$\begin{array}{l}\text { Gold standard* } \\
\qquad\left[\mathrm{cm}^{2}\right]\end{array}$} & \multicolumn{6}{|c|}{ Magnetic resonance imaging $\left[\mathrm{cm}^{2}\right]$} \\
\hline & & Axial & Difference & Sagittal & Difference & Coronal & Difference \\
\hline 1 & 13.24 & 10.50 & -2.74 & 11.32 & -1.92 & 10.77 & -2.47 \\
\hline 2 & 16.69 & 15.19 & -1.50 & 14.36 & -2.33 & 16.30 & -0.39 \\
\hline 3 & 22.45 & 16.02 & -6.43 & 18.51 & -3.94 & 19.89 & -2.56 \\
\hline 4 & 52.96 & 40.61 & -12.35 & 22.93 & -30.03 & 33.15 & -19.81 \\
\hline 5 & 56.41 & 42.27 & -14.14 & 25.69 & -30.72 & 41.72 & -14.69 \\
\hline Mean & 32.35 & 24.92 & -7.43 & 18.56 & -13.79 & 24.37 & -7.98 \\
\hline SD & 20.69 & 15.24 & 5.64 & 5.92 & 15.16 & 12.73 & 8.69 \\
\hline
\end{tabular}

*The surface area which is calculated by the physical section of the spleen was accepted as the gold standard; SD — standard deviation
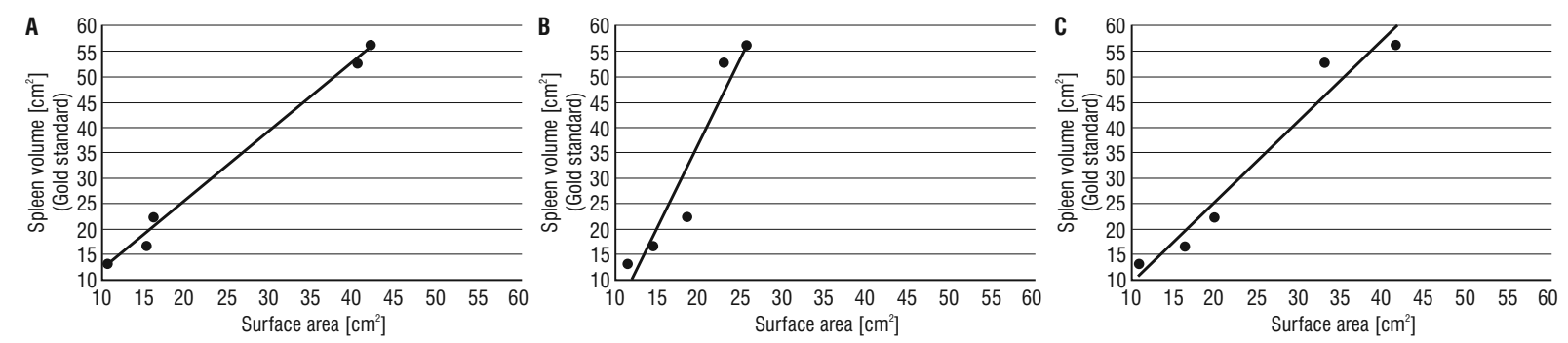

Figure 5. Scatter plots displaying the surface area estimates of gold standard versus (A) axial magnetic resonance imaging (MRI), (B) sagittal MRI, (C) coronal MRI.

in USG measurement and overestimation in caliper measurement. The mean \pm SD decrease in spleen volume for USG measurement was $-0.12 \pm 1.54 \mathrm{~cm}^{3}$ and the mean increase for caliper measurement was $1.16 \pm 1.97 \mathrm{~cm}^{3}$ (Table 3, Fig. 4).

We have found that volumes calculated by using physical section were closest to the gold standard (ICC = 0.99). In addition, volumes calculated in coronal MR planes showed closest values to the gold standard (ICC $=0.98)$. Pearson's correlation and $p$ values seem to support our results. Results for all methods are reported in Table 4.

We have estimated the mean \pm SD spleen surface area using cycloid probe on slices of cadavers as a $32.3 \pm 20.6 \mathrm{~cm}^{2}$. The surface area, which is calculated by the physical section of the spleen, was accepted as the gold standard. The surface areas estimated by cycloid probe using axial, coronal and sagittal MR images were $24.9 \pm 14.36 \mathrm{~cm}^{2}$, $24.3 \pm 12.7 \mathrm{~cm}^{2}$ and $18.5 \pm 5.9 \mathrm{~cm}^{2}$, respectively. There were no differences among all methods ( $p>0.05$ ) (Table 5).

We have determined underestimation of spleen surface area for axial, sagittal and coronal MR images.
The mean \pm SD differences in spleen surface area for axial, sagittal and coronal MR images were -7.43 \pm $\pm 5.64 \mathrm{~cm}^{3},-13.79 \pm 15.16 \mathrm{~cm}^{3}$ and $-7.98 \pm$ $\pm 8.69 \mathrm{~cm}^{3}$, respectively (Table 5 , Fig. 5 ).

According to Figure 5, we have compared the surface areas obtained by physical section and MRI images (axial, sagittal and coronal). As a result, the axial MRI based surface area calculation provided significantly closer values to the gold standard (CCC $=0.86, \mathrm{ICC}=0.95)$ than sagittal or coronal MRI based surface area calculation. The spleen surface area CCC statistics obtained with axial, sagittal, and coronal MRI were $0.86 \%, 0.33 \%, 0.77 \%$ in subjects, respectively. Pearson's correlation and $p$ values support our results (Table 6). Moreover, the mean CE of the spleen volumes and surface area are given in Table 7 .

\section{DISCUSSION}

Organ volume measurement plays an important role in the clinical diagnosis and follow-up of diseases. Many diseases present changes in size and morphology of the organs. Therefore, determining normal parameters of the organs bears great impor- 
Table 6. Statistical agreement measures for axial, sagittal and coronal magnetic resonance imaging

\begin{tabular}{lccc}
\hline Agreement measures & \multicolumn{3}{c}{ Magnetic resonance imaging [cm $\left.{ }^{2}\right]$} \\
\cline { 2 - 4 } & Axial & Sagittal & Coronal \\
\hline CCC $(95 \% \mathrm{CI})$ & $0.86(0.52-0.97)$ & $0.33(0.00-0.61)$ & $0.77(0.33-0.93)$ \\
ICC $(95 \% \mathrm{CI})$ & $0.95(0.62-0.99)$ & $0.50(0.00-0.93)$ & $0.87(0.21-0.99)$ \\
r (95\% Cl) & $0.99(0.95-1.00)$ & $0.95(0.44-1.00)$ & $0.98(0.68-1.00)$ \\
ppt & 0.042 & 0.112 & 0.109 \\
pls & $<0.001$ & 0.012 & 0.004 \\
CV (\%) & 22.19 & 53.72 & 27.79 \\
\hline
\end{tabular}

CCC — concordance correlation coefficient; $\mathrm{Cl}$ — confidence interval; ICC — intraclass correlation coefficient; $r$ - Pearson's correlation coefficient; ppt — $p$ value obtained from paired-t test; $\mathrm{pls}$ - $\mathrm{p}$ value obtained from least square analysis; $\mathrm{CV}$ - coefficient of variation; maximum CCC, ICC and $r$ values and minimum ppt, pls and CV values indicate best agreement between axial MRI and gold standard surface area

Table 7. The coefficient of error (CE) values for point-counting ( $\left.C E_{P C}\right)$, Cavalieri ( $\left.C E_{C A V}\right)$ and total $\left(C E_{T o t a l}\right)$ for spleen volume and surface area

\begin{tabular}{lcccc}
\hline Magnetic resonance imaging & \multicolumn{3}{c}{ CE volume [\%] } & $\begin{array}{c}\text { CE surface } \\
\text { area [\%] }\end{array}$ \\
\cline { 2 - 4 } & Cavalieri & Point-counting & Total & $2.94 \pm 0.50$ \\
\hline Axial & $2.57 \pm 2.13$ & $2.57 \pm 1.18$ & $3.76 \pm 2.20$ & $3.41 \pm 1.76$ \\
Sagittal & $1.83 \pm 1.62$ & $2.51 \pm 0.32$ & $3.30 \pm 1.55$ & $3.16 \pm 0.84$ \\
Coronal & $1.59 \pm 0.98$ & $2.71 \pm 0.44$ & $3.17 \pm 1.86$ & \\
\hline
\end{tabular}

tance in terms of accurate evaluation of pathologic changes [7].

In the literature, there are numerous studies on measurement of organ volumes by $\mathrm{CT}[2,26]$ and MRI [10]. Those studies have used Cavalieri's principle, a stereological method for organ volume measurement $[1,2,9]$. The volumes of organs such as heart [27], liver [33], prostate [3], ventricle [22], brain [24], and also the volume of foetus [29], have been measured using MR imaging and Cavalieri's principle.

There are many imaging methods used for spleen volume measurement. Among them, USG [4, 32], CT [26], MRI [21], nuclear scintigraphy [17], and conventional radiography [19] are the commonly applied ones. Most of these modalities have certain advantages and disadvantages concerning accurate volume measurement [21]. There are many studies about the sonographic evaluation of the spleen.

Ishibashi et al. [15] developed a splenic index based on 2 linear sonographic measurements and found a relationship between the volumes of the removed spleens and this index.

Rosenberg et al. [32] measured splenic length using USG in 230 infants and children. Accordingly, the mean splenic length in the $0-3$ month group $(n=28)$ was $4.5 \mathrm{~cm}$, with an upper range of $6 \mathrm{~cm}$. There was no difference in splenic length between the 2 genders until 15 years of age, however, males were reported to have a slightly larger spleen after that age. Megremis et al. [23] evaluated the relationship of splenic length measured by USG with age, gender, and somatometric parameters in 512 healthy children aged 0-17 years. Statistical analyses revealed that splenic length was correlated with the parameters of age, height, weight, and body surface area. The highest correlation was between splenic length and height, whereas there was no significant difference between the 2 genders with regard to splenic length. The mean splenic length was $4.40 \pm 0.50 \mathrm{~cm}$ in the $0-3$ month age group. In our study, the mean splenic length measured by USG was $3.29 \pm 1.09 \mathrm{~cm}$, whereas the mean splenic length measured by dividers on dissected spleens was $3.42 \pm 1.19 \mathrm{~cm}$. The low values found in our study can be attributed to the lower number of cases or mean age.

Ashgar et al. [4] aimed to establish normative data for splenic dimensions in North Indian population. They enrolled 21 patients (12 male and 9 female) aged 20-70 years with no spleen-related pathology. Splenic volume was measured by 2 methods. These methods are volume and surface rendering technique of able 3-D-Doctor software and ellipsoid formula. In 
our study, we have estimated splenic volume by USG based on ellipsoid formula as well.

Hidaka et al. [13] conducted a study on 30 healthy volunteers and 30 patients with cirrhosis, in which they calculated estimates of splenic volume using 3D USG and CT. USG measurements were performed by 2 experienced radiologists and the estimated values were calculated by using virtual organ computer-aided analysis (VOCAL). The mean splenic volume values obtained by USG were reported to be close to the ones obtained by CT. In this study, 3D USG measurement using VOCAL technique was reported to be a reliable, fast, and valid method for estimating splenic volume.

Yetter et al. [36] estimated splenic volume in 142 liver patients using helical CT and USG. In the end, USG measurements of spleen volume based on the ellipsoid formula were found to be more reliable. Downey [8] and Rodrigues et al. [31] also applied ellipsoid formula for the estimation of splenic volume. In our study, we have estimated splenic volume by USG based on ellipsoid formula as well.

Thayyil et al. [34] estimated the weight of internal organs by post-mortem MRI using non-invasive $3 D$ volumetric reconstruction method in foetuses, newborns and children. They determined a strong correlation between the actual and estimated weights of the organs. The estimates of foetuses younger than 20 weeks and lighter than $300 \mathrm{~g}$ were observed to show a decreasing trend of accuracy.

Mazonakis et al. [21] estimated splenic volume using random marking technique on MR images. They enrolled 16 adult patients (10 male and 6 female) with no spleen-related pathology. The images were transferred into computer environment and 2 readers separately calculated volumetric estimates using random marking and manual planimetry techniques. The random marking technique was found to be a reproducible and fast technique with a higher accuracy rate in the estimation of spleen volume.

In gold standard volumetric studies, which new volume estimation methods such as stereology, segmentation methods etc., were compared to fluid displacement technique, cadaveric specimens were reported to be necessary. The supply of cadavers is difficult, so many researchers, as we have mentioned below, used a limited number of cadaveric specimens. Similar to other studies, we have used 5 newborn cadavers. Sahin et al. [33] studied the liver volume using fluid displacement technique and stereological methods in MR images. They used 5 normal cadaveric livers. In addition, they did not find any significant differences between the estimation results of 2 methods ( $p>0.05$ ). Emirzeoglu et al. [9] investigated liver volume using CT scans. In their study, 5 normal livers obtained from cadavers were scanned using a Spiral CT Scanner in the horizontal plane. They compared results, which were obtained by the fluid displacement technique and stereological method. Acer et al. [3] performed volumetric measurements on the prostate glands, obtained after radical prostatectomy, of 5 adults using USG and Archimedes' principle. They employed ellipsoid formula for the volumetric calculation by USG.

A CE of $5 \%$ is regarded as appropriate for many organ measurements based on Cavalieri's principle [31]. In the literature, it is noted that at least 6-7 sections should be obtained from an organ and that the total number of points corresponding to the entire sections should not be below 100-200. As a result of this procedure, the coefficient of error is expected to drop below $5 \%[12,28]$. By applying such a procedure, the coefficient of error is maintained at or even below the desired values. In our study, the coefficient of error values obtained from the volume and surface area measurements were found to be below $5 \%$.

\section{CONCLUSIONS}

In the present study, the spleen volumes of the newborn cadavers were measured using USG, MRI, Archimedean, and slicing methods. In conclusion, we believe that the results of our study will contribute to the clinical evaluation and preoperative surgical planning of diseases that would change the spleen size.

\section{ACKNOWLEDGEMENTS}

This study was supported by the Department of Scientific Research Projects of Erciyes University.

We wish to thank Kazım Ziya Gümüş, Assistant Prof; Kenan Aycan, Prof. Dr; Erdogan Unur, Prof. Dr; Harun Ulger, Prof. Dr, for skillful technical assistance.

\section{REFERENCES}

1. Acer N, Cankaya MN, Işçi $\mathrm{O}$, Baş $\mathrm{O}$, Çamurdanoğlu M, Turgut M (2010) Estimation of cerebral surface area using vertical sectioning and magnetic resonance imaging: a stereological study. Brain Res, 1310: 29-36.

2. Acer N, Sahin B, Usanmaz M, Tatolu H, Irmak Z (2008) Comparison of point counting and planimetry methods for the assessment of cerebellar volume in human using 
magnetic resonance imaging: a stereological study. Surg Radiol Anat, 30: 335-339.

3. Acer N, Sofikerim M, Ertekin T, Unur E, Cay M, Oztürk F (2011) Assessment of in vivo calculation with ultrasonography compared to physical sections in vitro: a stereological study of prostate volumes. Anat Sci Int, 86: 78-85.

4. Asghar A, Agrawal D, Yunus SM, Sharma PK, Zaidi SHH, Sinha A (2011) Standard Splenic Volume Estimation in North Indian Adult Population: Using 3D Reconstruction of Abdominal CT Scan Images. Anat Res Int, 2011: 707325.

5. Baddeley AJ, Gundersen, HJ, Cruz-Orive LM (1986) Estimation of surface area from vertical sections. J. Microsc, 142: 259-276.

6. Corkill JA, Brabin BJ, MacGregor DF, Alpers MP, Milner RD (1989) Newborn splenic volumes vary under different malaria endemic conditions. Arch Dis Child, 64: 541-545.

7. Dogan TH, Basak M, Karatag O, Degirmenci H, Ozkurt H (2004) Evaluation of liver, spleen and kidney sizes by ultrasonography in normal children between the ages of 0-14. Turk J Pediatr, 47: 107-113.

8. Downey MT (1992) Estimation of splenic weight from ultrasonographic measurements. Can Assoc Radiol J, 43: 273-277.

9. Emirzeoğlu M, Sahin B, Selcuk MB, Kaplan S (2005) The effects of section thickness on the estimation of liver volume by the cavalieri principle using computed tomography images. Eur. Radiol, 56: 391-397.

10. Ertekin T, Acer N, Turgut AT, Aycan K, Ozçelik O, Turgut M (2011) Comparison of three methods for the estimation of the pituitary gland volume using magnetic resonance imaging: a stereological study. Pituitary, 14: 31-38.

11. Gundersen HJ, Jensen EB (1987) The efficiency of systematic sampling in stereology and its prediction. J Microsc, 147: 229-263.

12. Gundersen HJG, Jensen EBV, Kieu K, Nielsen J (1999) The efficiency of systematic sampling in stereology-reconsidered. J Microsc, 193: 199-211.

13. Hidaka $H$, Nakazawa $T$, Wang $G$, Kokubu $S$, Minamino $T$, Takada J, Tanaka Y, Okuwaki Y, Watanabe M, Shibuya A, Koizumi W (2010) Reliability and validity of splenic volume measurement by 3-D ultrasound. Hepatology Res, 40: 979-988.

14. I De Odorico, Spaulding KA, Pretorius DH, Lev-Toaff AS, Bailey TB, Nelson TR (1999) Normal splenic volumes estimated using three-dimensional ultrasonography. J Ultrasound Med, 18: 231-236.

15. Ishibashi $H$, Higuchi N, Shimamura R, Hirata $Y$, Kudo J, Niho $Y$ (1991) Sonographic assessment and grading of spleen size. J Clin Ultrasound, 19: 21-25.

16. Junqueira LC, Carneiro J, Kelley RO (2003) Basic histology. McGraw Hill Companies, Sao Paulo, Brazil, pp. 284-288.

17. Konus OL, Ozdemir A, Akkaya A, Erbas G, Celik H, Isik S (1998) Normal liver, spleen, and kidney dimensions in neonates, infants, and children: evaluation with sonography. AJR, 171: 1693-1698.

18. Lin L, Hedayat AS, Sinha B, Yang M (2002) Statistical methods in assessing agreement: models, issues, and tools. J Am Stat Assoc, 97: 257-270.

19. Loftus WK, Chow LT, Metreweli C (1999) Sonographic measurement of splenic length: correlation with measurement at autopsy. J Clin Ultrasound, 27: 71-74.

20. Mayhew TM, Olsen DR (1991) Magnetic resonance imaging (MRI) and model-free estimates of brain volume determined using the Cavalieri principle. J Anat, 178: 133-144.

21. Mazonakis $M$, Damilakis J, Maris T, Prassopoulos $P$, Gourtsoyiannis N (2000) Estimation of spleen volume using MR imaging a random marking technique. Eur Radiol, 10: 1899-1903.

22. Mazonakis M, Pagonidis K, Damilakis J (2011) Right ventricular volumes and ejection fraction by MR imaging and stereology: comparison with standard image analysis method. Clin Anat, 24: 868-873.

23. Megremis SD, Vlachonikolis IG, Tsilimigaki AM (2004) Spleen lenght in childhood with us: normal values based on age, sex, and somatometric parameters. Radiology, 231: 129-134.

24. Nisari M, Ertekin T, Ozcelik O, Cinar S, Doganay S, Acer N (2012) Stereological evaluation of the volume and volume fraction of newborns' brain compartment and brain in magnetic resonance images. Surg Radiol Anat, 34: 825-832.

25. Pakkenberg B (1992) Stereological quantitation of human brains from normal and schizophrenic individuals. Acta Neurol Scand Suppl, 137: 20-33.

26. Prassopoulos P, Daskalogiannaki M, Raissaki M, Hatjidakis A, Gourtsoyiannis N (1997) Determination of normal splenic volume on computed tomography in relation to age, gender and body habitus. Eur Radiol, 7: $246-248$

27. Roberts N, Cruz-Orive LM, Bourne M, Herfkens RJ, Karwoski RA, Whitehouse GH (1997) Analysis of cardiac function by MRI and stereology. J Microsc, 187: 31-42.

28. Roberts N, Cruz-Orive LM, Reid NM, Brodie DA, Bourne M, Edwards RH (1993) Unbiased estimation of human body composition by the Cavalieri method using magnetic resonance imaging. J Microsc, 171: 239-253.

29. Roberts N, Garden AS, Cruz-Orive LM. Whitehouse GH. Edwards RH (1994) Estimation of fetal volume by magnetic resonance imaging and stereology. $\mathrm{Br} J$ Radiol, 67: 1067-1077.

30. Roberts N, Puddephat MJ, McNulty V (2000) The benefit of stereology for quantitative radiology. $\mathrm{Br} J$ Radiol, 73: 679-697.

31. Rodrigues AJ, Rodrigues CJ, Germano MA, Rasera I, Cerri GG (1995) Sonographic assessment of normal spleen volume. Clin Anat, 8: 252-255.

32. Rosenberg HK, Markowitz RI, Kolberg H, Park C, Hubbard A, Bellah RD (1991) Normal splenic size in infants and children: sonographic measurements. AJR, 157:119-121.

33. Sahin B, Ergur H (2006) Assessment of the optimum section thickness for the estimation of liver volume using magnetic resonance images: a stereological gold standard study. Eur J Radiol, 57: 96-101.

34. Thayyil S, Schievanoa S, Robertson NJ, Jones R, Chitty LS, Sebire NJ, Taylor AM; MaRIAS (Magnetic Resonance Imaging Autopsy Study) Collaborative group (2009) A semi-automated method for non-invasive internal organ weight estimation by post-mortem magnetic resonance imaging in fetuses, newborns and children. Eur. Radiol, 72: $321-326$.

35. Thompson RB (1977) Disorders of the blood. A textbook of clinical haematology. Churchill Livingstone, London.

36. Yetter EM, Acosta KB, Olson MC, Blundell K (2003) Estimating splenic volume: sonographic measurements correlated with helical CT determination. AJR, 181: 1615-1620. 